\title{
Liderazgo Directivo en Escuelas que Superan las Barreras del Contexto
}

\section{Principal Leadership in Schools that Overcome Contextual Barriers}

\author{
Claudia Romero * \\ Universidad Torcuato Di Tella, Argentina
}

\begin{abstract}
DESCRIPTORES:
Liderazgo directivo

Eficacia escolar

Equidad

Calidad

Escuela

RESUMEN:

Los directivos escolares son factores clave en el logro de buenas escuelas y esto es especialmente relevante en contextos de desigualdad como América Latina en general y Argentina en particular. El objetivo de investigación es comprender de qué manera el liderazgo directivo escolar opera como factor de equidad y eficacia en escuelas. Se adopta un enfoque cualitativo, de estudio de casos múltiples, centrado en las prácticas de directivos de cuatro escuelas secundarias públicas de la Ciudad de Buenos Aires. Para la selección de escuelas se toman los resultados del Índice de Equidad y Calidad de la Educación Porteña (IECEP) elaborado por el Ministerio de Educación de la Ciudad de Buenos Aires para sus escuelas secundarias y como indicador de vulnerabilidad educativa se considera el nivel educativo de la madre promedio por escuela, calculado por el mismo organismo. Los resultados aportan concepciones y prácticas del liderazgo directivo, que consisten en sostener una visión crítica y reflexiva sobre los problemas de desigualdad, construir un proyecto compartido con sentido de comunidad, mantener altas expectativas y compromiso pedagógico y promover estrategias equitativas y de inclusión. Los hallazgos son consistentes con la literatura de investigación sobre liderazgo pedagógico, inclusivo y distribuido. Las conclusiones realizan un aporte para las políticas educativas de desarrollo del liderazgo directivo, destinadas al logro de sistemas educativos de mayor calidad y más justos.
\end{abstract}

\section{KEYWORDS:}

School leadership

School effectiveness

Equity

Quality

Schools

\begin{abstract}
:
School leaders are key factors in achieving good schools and this is especially relevant in contexts of inequality such as Latin America in general and Argentina in particular. The research objective is to understand how school leadership operates as a factor of equity and effectiveness in schools. A qualitative approach is taken of a multiple case study focused on the practices of the directors of four public high schools in the City of Buenos Aires. For the selection of schools, the results base is taken from the Index of Equity and Quality of Buenos Aires Education (IECEP) prepared by the Ministry of Education of the City of Buenos Aires for its secondary schools and as an indicator of educational vulnerability, the educational level of the average mother per school is considered calculated by the same ministry.The results provide effective leadership conceptions and practices developed in these schools, which consist of sustaining a critical and reflective vision around the problems of inequality, building a shared project with a sense of community, maintaining high expectations and pedagogical commitment, and promoting equitable and inclusive strategies. The findings are consistent with the research literature on pedagogical, inclusive and distributed leadership. The research contributes to educational policies for the development of school leadership, aimed at achieving higher quality and fairer educational systems.
\end{abstract}

CÓMO CITAR:

Romero, C. (2021). Liderazgo directivo en escuelas que superan las barreras del contexto. REICE. Revista Iberoamericana sobre Calidad, Eficacia y Cambio en Educación, 19(1), 73-90. https://doi.org/10.15366/reice2021.19.1.005

*Contacto: claudiaromero@utdt.edu

ISSN: $1696-4713$

www.rinace.net/reice/

revistas.uam.es/reice
Recibido:

5 de abril 2020

1a Evaluación: 28 de junio 2020

$2^{\text {a }}$ Evaluación: 10 de julio 2020

Aceptado: 25 de julio 2020 


\section{Introducción}

América Latina es una región de profundas desigualdades cuyos sistemas educativos tienden a reproducirlas y legitimarlas a través de diversos procesos, entre ellos la creciente segregación escolar por la cual los estudiantes más vulnerables tienden a concentrarse en ciertas escuelas y los más favorecidos en otras. Este mecanismo puede potenciar las desventajas de origen y reforzar la exclusión de los grupos más desfavorecidos sobre los cuales pesa la profecía auto cumplida de que obtendrán menos educación y de peor calidad.

En ese contexto, como muestran las investigaciones, las escuelas, en presencia de determinados factores, en particular el liderazgo directivo, pueden agregar valor y revertir las determinaciones de origen. Si bien en términos ideales, toda escuela está llamada a producir aprendizajes de calidad con equidad, pocas lo logran. De allí que un importante desafío para las políticas de lucha contra las desigualdades sea la identificación de liderazgos en "buenas escuelas" (Romero, 2018), escuelas justas o "escuelas resilientes" (Day y Gu, 2016), es decir aquellas que logran contrarrestar las inequidades, con el fin de comprender sus prácticas en la construcción de una cultura escolar de calidad, más equitativa y justa.

En este estudio se procura comprender las características del liderazgo directivo en el desarrollo de "buenas escuelas" que atienden alumnado con alta vulnerabilidad que logran superar las barreras del contexto. La investigación de tipo cualitativa de estudio de casos múltiples se realiza a directivos de cuatro escuelas secundarias públicas de la Ciudad de Buenos Aires que cuentan con la doble condición de población vulnerable con una situación inicial desaventajada y que, sin embargo, obtienen resultados de aprendizaje por encima del promedio de la Ciudad.

¿De qué manera el liderazgo directivo promueve el logro de escuelas capaces de revertir las determinaciones de la cuna? ¿Qué visiones y prácticas orientan el accionar de estos directivos? y en definitiva ¿Cuál es el liderazgo a fomentar por las políticas que luchan contra la desigualdad?

\section{Revisión de la literatura}

El liderazgo directivo es un factor clave para hacer de una escuela, una buena escuela (Romero, 2018). En efecto, las escuelas pueden ser lugares de reproducción y legitimación de desigualdades sociales o formidables ámbitos de construcción de prácticas contraculturales que contribuyan a producir calidad con equidad y justicia. Y, como muestran las investigaciones, el liderazgo directivo puede hacer una diferencia. Cuando las sombras de la pobreza, la desigualdad y la exclusión cubren a las sociedades, como es el caso de América Latina, la escuela y el liderazgo directivo, importan.

\subsection{Inequidad educativa y segregación escolar en América Latina y Argentina}

América Latina en su territorio de desigualdades, presenta altos niveles de segregación en relación con el resto del mundo y Argentina se ubica en una posición de moderada a alta tanto en el nivel primario como secundario (Krüger, 2019a; Murillo y Martínez-Garrido, 2017; Vázquez, 2012) y la Ciudad de Buenos Aires está entre las jurisdicciones que se destaca por sus mayores niveles de segregación (Krüger, 2019b). Por su parte, los estudios que analizan para América Latina la relación entre nivel socioeconómico de las familias y elección de escuelas muestran que Argentina, junto a Brasil, son los países que tienen mayor diferencia social entre quienes acuden a escuelas privadas y escuelas de gestión pública y que la segregación público-privada por nivel socioeconómico en la región va en aumento tanto para el nivel primario como secundario (Arcidiácono, 2014; Pereyra, 2008).

En América Latina existe evidencia sistemática que ratifica que cuanto más pobre y vulnerable socialmente es el alumnado, menores son las probabilidades de permanecer en la escuela y de alcanzar buenos desempeños (Román, 2013). Las escuelas públicas son también las que, en Argentina, obtienen resultados de aprendizaje más bajos en las pruebas internacionales y nacionales generándose así circuitos de calidad escolar diferenciados que se acoplan a la segregación socioeconómica (Krüger, 2019b). Y como señala Román (2013), la deserción y los bajos logros de aprendizaje ter- 
minan siendo en gran medida "algo construido desde la propia escuela, a partir de sus dinámicas, juicios, prejuicios y prácticas” (p. 34)

Para el caso de Argentina el estudio realizado por Romero, Krichesky y Zacarías (2012) en el nivel secundario, que es al que pertenecen los directivos que estudiamos, expone como, a pesar de la expansión del nivel que es obligatorio desde 2006, crecen los índices de repitencia, abandono y bajo desempeño, que se explican por la persistencia de una cultura y estructura organizacional selectiva y una matriz curricular academicista. El propio diseño de la escuela secundaria representa una invitación al fracaso de los sectores más vulnerables.

\subsection{Aportes de la investigación sobre Eficacia Escolar: el factor escuela y el liderazgo directivo}

La investigación sobre Eficacia Escolar (School Effectiveness) que estudia la incidencia que distintos aspectos intraescolares tienen en los logros de aprendizaje de los estudiantes, ha realizado aportes relevantes acerca del valor del factor escuela y en particular del liderazgo directivo en el logro de escuelas eficaces o "buenas escuelas". Esta línea de investigación guarda estrecha relación con los principios de equidad y justicia social desde sus comienzos. En una de las principales obras, Mortimore (1991) define a una escuela eficaz como "aquella en la que los alumnos progresan a un nivel mayor del que podría esperarse según sus condiciones de entrada o base” (p. 9). El concepto de eficacia queda implícito en cualquier definición aceptable de lo que llamamos "buena escuela", más allá de las características específicas que conforman la cultura escolar (Murillo, 2007). De modo que una buena escuela lo es, en principio, si resulta eficaz.

La investigación sobre Eficacia Escolar en América Latina y el Caribe (Murillo, 2008) define como factores asociados al desarrollo del alumnado: a) el sentido de comunidad, b) clima escolar y de aula, c) dirección escolar, d) currículo de calidad, e) gestión del tiempo, f) participación de la comunidad escolar, g) desarrollo profesional de los docentes, y h) altas expectativas y i) instalaciones y recursos. Es cierto que cada factor de manera aislada tiene impacto limitado y es en realidad su combinación integral, la que configura una cultura orientada a la eficacia (Bolívar, 1999).

En cuanto al liderazgo directivo, las investigaciones lo señalan como el segundo factor intraescolar cuyo efecto indirecto en el logro de aprendizajes procede promoviendo un clima escolar de estímulo y motivación al profesorado y garantizando condiciones de trabajo y desarrollo profesional (Hargreaves y Fullan, 2012; Leithwood et al., 2006).

\subsection{El liderazgo directivo vinculado a la inclusión y la equidad}

La literatura indica que el liderazgo directivo tiene efectos mayores en escuelas de contextos vulnerables, (Leithwood et al., 2004) constituyéndose en un factor también de equidad (Murillo et al., 2010; Romero, 2018; Weinstein, 2017). Los directivos de escuelas con poblaciones vulnerables que obtienen buenos resultados de aprendizaje desarrollan estrategias de apoyo a los estudiantes que tienen bajos logros, promueven participación de las familias y aseguran distribución prioritaria de recursos para el alumnado con mayores necesidades (Field, Kuczeram y Pont, 2007). En contextos de desigualdad, como los latinoamericanos, el liderazgo directivo implica asumir posiciones contraculturales. Esto supone además desarrollar una visión crítica sobre la escolarización, operar sobre prácticas inequitativas y estimular una cultura de inclusión (Ainscow y Kugelmass, 2005; Ryan, 2006).

El liderazgo de una buena escuela ha de ser pues un liderazgo crítico e inclusivo (Ryan, 2006, 2016) y compartido o distribuido (Spillane, 2006, 2013), a la vez que un "liderazgo pedagógico" (Bolívar, 2019), centrado en el alumnado y los aprendizajes, que permita construir comunidades de aprendizaje, generar capacidades y desarrollo endógeno en la consecución de mayores niveles de calidad y equidad. En los últimos años se sintetizan todos estos elementos, con énfasis en los procesos de redistribución y reconocimiento de recursos y oportunidades, en la idea del "liderazgo para la justicia social” (Belavi y Murillo, 2020; Murillo y Hernández, 2014). 


\subsection{Más que modelos, prácticas de liderazgo}

Un giro se ha producido en la última década en la investigación sobre liderazgo directivo que en términos de Bolívar puede resumirse como "pasar de los modelos a las prácticas” (Bolívar, 2019) En efecto, la investigación estuvo centrada en delinear estilos o modelos, algunos de los cuales acabamos de revisar: "liderazgo pedagógico", "inclusivo", "distribuido", pero dado que el liderazgo es situacional y responde a un contexto (Day y Sammons, 2011) lo que parece ser realmente significativo a la hora de establecer relaciones con el desempeño de los estudiantes son determinadas "buenas prácticas” o prácticas exitosas de liderazgo (Louis, 2017).

En este sentido, se destacan los trabajos de Robinson $(2014,2017)$ que vinculan liderazgo con resultados de los estudiantes, al identificar cinco prácticas de liderazgo que lo hacen eficaz: 1) definir objetivos y expectativas, 2) garantizar enseñanza de calidad, 3) asignar estratégicamente los recursos, 4) promover el desarrollo profesional del cuerpo docente y 5) asegurar entornos ordenados y de apoyo. Teniendo en cuenta lo expuesto y como definición conceptual de la investigación se considera al liderazgo directivo eficaz y equitativo en escuelas con población vulnerable como el conjunto de prácticas contextualizadas de carácter pedagógico, distribuido e inclusivo capaces de producir niveles de calidad y equidad mayores a los que son esperables para esa población.

Con estos antecedentes en esta investigación se plantea el objetivo general de comprender de qué manera las prácticas del liderazgo escolar operan como factor de eficacia y de equidad en cuatro escuelas secundarias de la Ciudad de Buenos Aires cuyos estudiantes obtienes mejores resultados que el promedio aun cuando pertenecen a sectores desaventajados. Para ello y como objetivos específicos, se ha definido:

- Identificar, describir y analizar las características del liderazgo en cada escuela.

- Comparar las características de los equipos directivos en las distintas escuelas.

Se espera que los resultados obtenidos contribuyan a establecer líneas de políticas referidas a la formación y ejercicio del liderazgo directivo como contribución a mitigar la desigualdad y la segregación escolar.

\section{Método}

La investigación se enmarca en una metodología cualitativa de estudio de casos múltiples en una muestra de cuatro escuelas secundarias públicas de la Ciudad de Buenos Aires. Se seleccionó el nivel secundario por su carácter tradicionalmente selectivo e inequitativo (Romero, Krichesky y Zacarías, 2012) a pesar de ser obligatorio en el país desde 2006.

Para la selección de los casos fue preciso identificar previamente escuelas en las que existiera evidencia de su eficacia, es decir que cumplieran con la doble condición de atender a población vulnerable y obtener buenos resultados de aprendizaje. Esta selección fue realizada cuidadosamente ya que constituye un elemento central del diseño metodológico de la investigación.

El camino seguido fue primeramente explorar la base de resultados del Índice de Equidad y Calidad de la Educación Porteña (IECEP) elaborado por la Dirección General de Evaluación de la Calidad Educativa del Ministerio de Educación de la Ciudad Autónoma de Buenos Aires para el nivel secundario.

El IECEP fue medido durante el año $2013^{1}$ en 300 escuelas secundarias de la Ciudad de Buenos Aires, de gestión pública y privada, con el afán de obtener una medida multidimensional del desempeño de las escuelas para categorizar los centros educativos según patrones comunes extra o intraescolares. El IECEP se compone de tres dimensiones y 8 indicadores (cuadro 1).

\footnotetext{
${ }^{1}$ Este es el último año del que se dispone información del Indice dado que el Ministerio de Educación dejo de producir el índice.
} 
Cuadro 1

Dimensiones e indicadores del IECEP

\begin{tabular}{|c|c|c|}
\hline Dimensión & \multicolumn{2}{|l|}{ Indicador } \\
\hline Eficiencia interna & Tasa de promoción & $\begin{array}{l}\text { Complemento de la tasa de } \\
\text { Repitencia }\end{array}$ \\
\hline $\begin{array}{l}\text { Desempeño aca- } \\
\text { démico }\end{array}$ & $\begin{array}{l}\text { Porcentaje de alumnado de cada escuela que } \\
\text { obtuvo resultados medio y alto en los niveles de } \\
\text { desempeño del Operativo Nacional Evaluación de } \\
\text { la Calidad (ONE) } 2010 \text { en Lengua/Matemática }\end{array}$ & $\begin{array}{l}\text { Diferencia entre el porcentaje de } \\
\text { alumnado del nivel alto en las prue- } \\
\text { bas ONE en } \\
\text { Lengua/Matemática y el del nivel } \\
\text { medio }\end{array}$ \\
\hline $\begin{array}{l}\text { Equidad de los } \\
\text { aprendizajes }\end{array}$ & \multicolumn{2}{|c|}{$\begin{array}{l}\text { Diferencia entre los resultados de los aprendizajes de una escuela (del ONE 2010) y el pro- } \\
\text { medio de los resultados del total de la Ciudad (del ONE 2010). }\end{array}$} \\
\hline
\end{tabular}

Nota. Elaboración propia a partir de Montoya (2013).

El IECEP es una fórmula estadística que resulta de la sumatoria de los 8 indicadores cuyos valores ponderados suman 10 y sirve de insumo para conocer en qué situación está cada escuela y, en consecuencia, hacia dónde deben orientarse los esfuerzos de mejora.

A su vez la Dirección General de Evaluación de la Calidad Educativa proporcionó la información sobre el promedio por escuela del nivel educativo de la madre, que fue utilizado en este caso como un indicador para aproximarnos a una medida de la situación de vulnerabilidad educativa de la población escolar de las escuelas. Esta decisión obedece a que, no existe otro indicador mejor entre la información estadística oficial disponible y que la investigación sobre Eficacia Escolar ha demostrado que el nivel de estudios de la madre suele ser el indicador de nivel cultural con mayor poder explicativo del rendimiento de los estudiantes (Carvallo, 2006; Murillo, 2008).

Se procedió luego a cruzar los resultados IECEP con el indicador de vulnerabilidad educativa (nivel educativo de la madre promedio por escuela) con el fin de identificar si existían escuelas que cumplieran con la doble condición de resultados de aprendizaje por encima del promedio y nivel de vulnerabilidad educativa por debajo del promedio.

De las 300 escuelas evaluadas por el IECEP, sólo 18 de ellas (12,5\%) cumplían con la doble condición que buscábamos: obtuvieron un índice IECEP por encima del promedio de la Ciudad y población con nivel educativo de la madre por debajo del promedio. De ese grupo de escuelas se seleccionaron las cuatro escuelas con menor promedio de nivel educativo de la madre, es decir que según este indicador se presentaban con mayor vulnerabilidad educativa. Resultaron ser las cuatro escuelas de gestión estatal, dos Bachilleres con orientación comercial que denominamos $\mathrm{C} 1$ y C2 y dos Escuelas Técnicas que denominamos T1 y T2.

Efectivamente se cumplen para la selección las dos condiciones buscadas. El IECEP promedio de la Ciudad para ese año fue de 6,1 y el promedio de las escuelas públicas fue de 4,9, todas las escuelas de la muestra superan el promedio de la Ciudad y el de su comuna, superando también el promedio de las escuelas estatales. El promedio del nivel educativo de la madre a nivel de la Ciudad fue de 5.52 y todas las escuelas se encuentran debajo del mismo (cuadro 2).

Cuadro 2

IECEP y vulnerabilidad educativa por cada escuela comparado con promedios de la comuna, y de la Ciudad (promedio)

\begin{tabular}{|c|c|c|c|c|c|c|}
\hline & \multicolumn{4}{|c|}{ IECEP } & \multicolumn{2}{|c|}{ Vulnerabilidad educativa } \\
\hline & \multirow{2}{*}{ Escuela } & \multirow{2}{*}{ Comuna } & \multicolumn{2}{|c|}{ Ciudad } & \multirow{2}{*}{ Escuela } & \multirow{2}{*}{ Ciudad } \\
\hline & & & Priv. + Estatal & Estatal & & \\
\hline Escuela C1 & 6,99 & 6,2 & \multirow{4}{*}{6,1} & \multirow{4}{*}{4,9} & 4,58 & \multirow{4}{*}{5,52} \\
\hline Escuela C2 & 6,65 & 6,3 & & & 4,95 & \\
\hline Escuela T1 & 6,68 & 5,8 & & & 4,69 & \\
\hline Escuela T2 & 6,62 & 6,2 & & & 4,85 & \\
\hline
\end{tabular}

Nota. Elaboración propia. 
Una vez seleccionadas las escuelas se confirmó en entrevistas con los supervisores de cada distrito su visión acerca de si estas cuatro escuelas podrían ser consideradas "buenas escuelas" en los términos en que han sido definidas y en los cuatro casos, desde la perspectiva de los supervisores que desconocían los resultados IECEP, se confirmó que resultaban elegibles para el estudio. Por último, se realizó un estudio preliminar exploratorio en las cuatro escuelas a fin de conocer el contexto de cada (Romero, 2019).

En síntesis, la selección de las escuelas cuyo liderazgo directivo nos proponemos estudiar se basó en tres procedimientos: cruzar resultados del IECEP con el indicador de "nivel educativo de la madre promedio por escuela" provisto por el Ministerio como proxy de vulnerabilidad educativa, corroboración de la selección con supervisores y estudio preliminar del contexto de cada escuela (figura 1)

Figura 1

Procedimiento para la selección de los casos de estudio

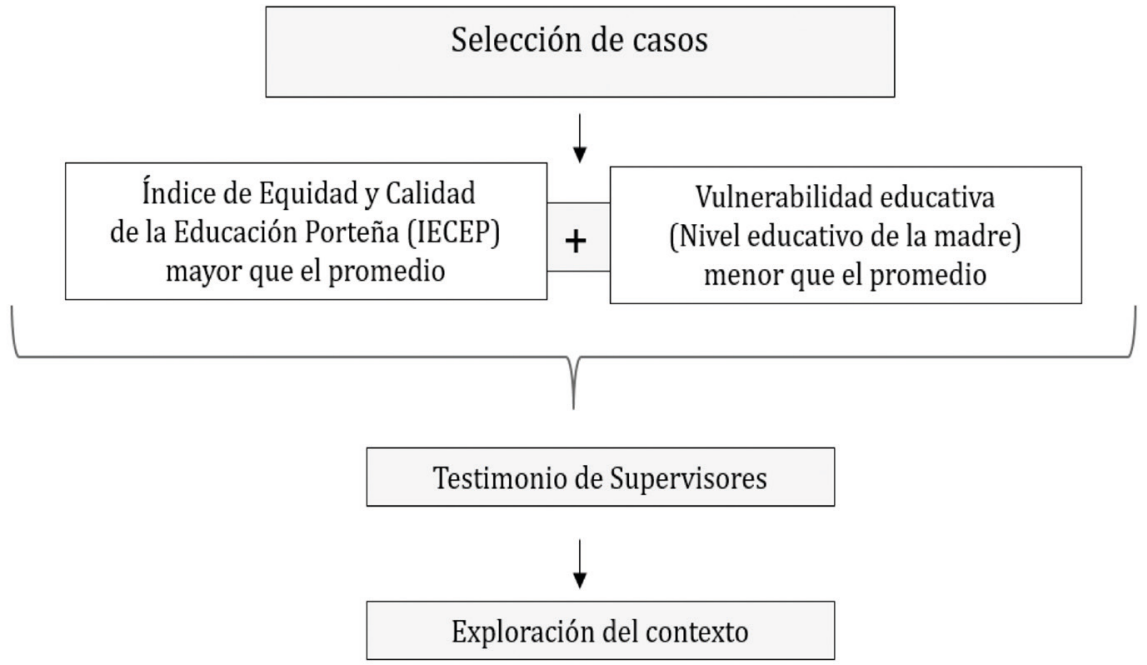

Nota. Elaboración propia.

Las cuatro escuelas ${ }^{2}$ están ubicadas en barrios de clase media: tres de ellas están próximas a asentamientos y barrios de emergencia y cercanas al límite que separa la Ciudad con el conurbano de la Provincia de Buenos Aires. Una tiene una ubicación más céntrica y posee buenos accesos desde distintos puntos de la Ciudad y cercana a terminales de tren y ómnibus provenientes también del conurbano. Dos de ellas son escuelas con orientación técnica y dos con orientación comercial. Son muy diferentes entre sí en cuanto al tamaño que va de los 1278 estudiantes a los 450 estudiantes. Tres de las escuelas tienen tres turnos (mañana, tarde y noche) y una sólo dos (mañana y tarde) (figura 2).

Una vez seleccionados los casos con los procedimientos expuestos, se emprendió el estudio propiamente dicho para dar cumplimiento a los objetivos propuestos. Se utilizó como instrumento la entrevista en profundidad a los cuatro directivos siguiendo el modelo de "encuentros reiterados cara a cara entre el investigador y los informantes" (Taylor y Bogdan, 1990, p. 101). Co ello se pretende comprenderlas perspectivas de los informantes respecto de sus experiencias o situaciones tal como las expresan con sus propias palabras. Se realizó una entrevista en profundidad a cada uno de los directivos de las cuatro escuelas en dos sesiones cada una. En la primera sesión se abordaron dos ejes temáticos: a) Biografía y experiencia formativa y profesional y b) Visión acerca de la educación escolar y sus principales problemas. En la segunda sesión se abordaron otros dos ejes temáticos: c) Proyecto institucional y d) Prácticas y agenda del director. Las entrevistas fueron grabadas y transcriptas y se realizó una codificación posterior para el análisis posterior.

\footnotetext{
${ }^{2}$ Se preserva la identidad de las escuelas en cumplimiento del marco legal vigente que rige en Argentina sobre la publicación de información relativa a resultados escolares.
} 
Figura 2

Ubicación de las escuelas de la muestra en la Ciudad Autónoma de Buenos Aires con IECEP por comuna, orientación académica, cantidad de alumnado, turnos y puntaje IECEP obtenido

\section{Muestra}

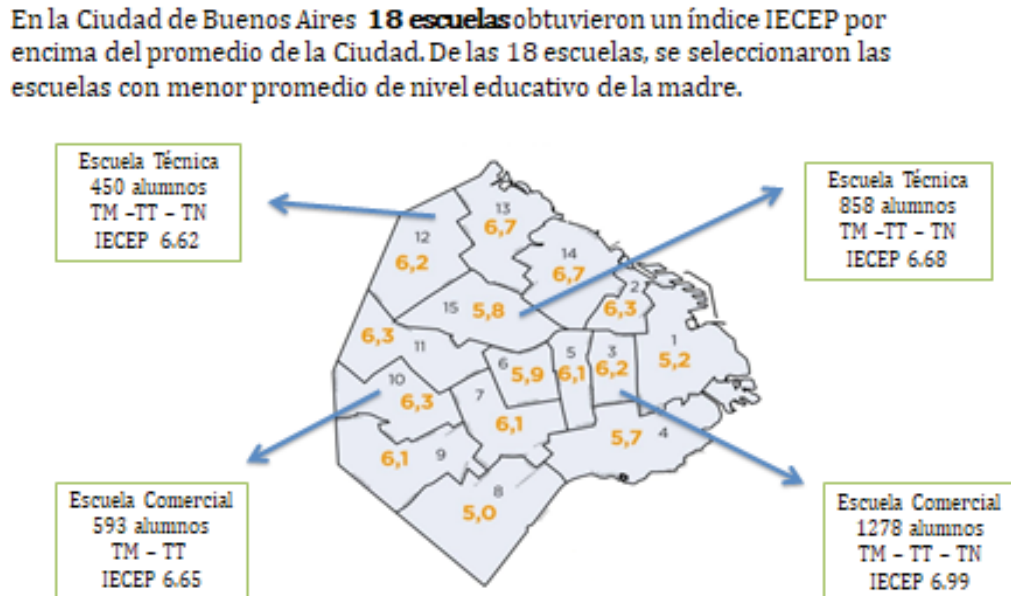

Nota. Elaboración propia.

El análisis de la información producida por las entrevistas responde a los parámetros procedimentales de la Teoría Fundamentada y, siguiendo los principales aportes de la literatura y nuestros objetivos de investigación, se definieron cuatro categorías de análisis:

- Visión crítica y reflexiva: posee una visión crítica de la escolarización que reproduce desigualdades, es sensible a situaciones de injusticia y discriminación y promueve la reflexión colectiva desarrollando un liderazgo inclusivo.

- Proyecto compartido y sentido de comunidad: conforma equipo directivo y liderazgo distribuido, comunidad profesional y fluido intercambio con familias y entornos. un proyecto compartido y alienta un clima de convivencia positivo y participativo.

- Altas expectativas y compromiso pedagógico: prioriza la función pedagógica de la escuela, mantiene altas expectativas respecto de estudiantes y docentes, desarrolla liderazgo pedagógico.

- Prácticas de promoción de la inclusión y la equidad: lleva adelante prácticas que remueven situaciones inequitativas, fomenta y desarrolla estrategias de apoyo y acompañamiento a los estudiantes.

\section{Resultados}

Los hallazgos de esta investigación se presentan siguiendo los objetivos propuestos; inicialmente se describe al directivo en el contexto de cada escuela y luego se presentan los resultados para cada categoría de análisis establecidas en el diseño metodológico.

\subsection{El directivo en el contexto de cada escuela}

La escuela $\mathrm{C} 1$ es un Bachillerato con Orientación Comercial, localizada en el centro de la Ciudad, de fácil acceso, lo cual habilita a que asistan estudiantes provenientes de la periferia y de la Provincia de Buenos Aires. La población escolar proviene en importante grado de sectores socioeconómicos de bajos recursos, muchos de ellos habitan en hoteles o pensiones; también y en menor medida hay alumnado de sector 
medio que reside en la zona. Actualmente la escuela cuenta con 1.278 estudiantes en los tres turnos. En los últimos tres años se ha logrado disminuir significativamente el porcentaje de abandono y la matrícula inicial se mantiene en cifras elevadas. El equipo de conducción está conformado por la rectora y un vicerrector por turno. Cuenta con Equipo de Orientación Escolar, Jefes de Departamento y un cuerpo de tutores.

$\mathrm{Marta}^{3}$ es la rectora desde hace 4 años y es profesora de la escuela desde hace 11 años. Es Licenciada en Gestión Educativa. El año anterior a la entrevista ganó la beca Fullbright de intercambio de directivos y visitó una escuela en Seattle, valora mucho esa experiencia como instancia de aprendizaje y de abrir su perspectiva sobre el liderazgo.

\begin{abstract}
Asumi el cargo que había dejado una directora de muchos años que se jubiló. Era un ícono. Yo decía, cómo voy a hacer para tener autoridad y mi propio estilo. Terminé la Licenciatura justo antes de asumir, eso me dio confianza y además pude armar un buen equipo, aunque tenemos nuestros estilos diferentes.... Trabajo usando mucho la información estadística que me permiten hacer seguimiento de cada grupo...La principal fortaleza de la escuela es su historia, su edificio, los buenos profesores, pero también la historia a veces nos juega en contra... la secundaria tradicional no nos sirve más, la escuela tiene que tener orden para trabajar, pero no puede ser expulsiva. (C1)
\end{abstract}

La escuela C2 es un Bachillerato con Orientación Comercial, ubicada en la zona de Montecastro, un barrio de casas bajas y alberga actualmente a 593 estudiantes, en dos turnos, mañana y tarde. Históricamente recibía a familias de clase media de la zona, pero en los últimos años la población ha cambiado y se acercan estudiantes de familias de menores recursos. La escuela mantiene una tasa de promoción por encima del 70\% a lo largo de los últimos tres años con un notable descenso del porcentaje de abandono. El equipo directivo está compuesto por la directora y una vicedirectora asignada al turno tarde. La institución cuenta Equipo de Orientación Escolar, cuerpo de tutores y Jefes de Departamento.

Leticia es la rectora hace 6 años, pero lleva una vida dentro de la escuela. Es Licenciada en Gestión Educativa y cursa una Maestría. Si bien su cargo está designado en el turno mañana asiste algunos días por la tarde para sostener contacto con el profesorado y estudiantes de ese turno.

Yo fui alumna, preceptora, jefe de preceptores y profesora de esta escuela, tengo un enorme cariño por ella. Fui profesora en otra escuela así que también pude verla desde afuera, eso es importante.... La escuela fue cambiando y yo también por suerte fui cambiando... la escuela pasó por distintos momentos, pero algo tiene que uno se quiere quedar, hay muchos profesores que son ex alumnos como yo, pero no diría que somos una familia, porque buscamos formarnos como profesionales, ser creativos...la escuela es más un lugar de pertenencia que nos desafía. (C2)

La escuela T1 es una Escuela con Orientación Técnica y está ubicada en el barrio de Chacarita, zona de desarrollo comercial que se destaca como nudo de la red de transportes de la Ciudad. Cuenta con muy buena infraestructura edilicia y equipamiento. Su matrícula es de 858 estudiantes y tiene tres turnos (mañana, tarde y noche) Los estudiantes pertenecen tradicionalmente a familias de clase media baja y en los últimos 10 años se incorporaron estudiantes de barrios vulnerables cercanos. Un 30\% de estudiantes pertenecen a familias de países limítrofes (Bolivia, Perú y Paraguay). La escuela evidencia disminución significativa del porcentaje de estudiantes repitentes en los últimos años, así como también es muy bajo el porcentaje de estudiantes que abandonan (1\%). El equipo directivo está conformado por la rectora, un vicedirector por turno, un regente. Tiene asesora pedagógica, cuerpo de tutores y Jefes de Departamentos y Talleres.

Blanca es la rectora desde hace 4 años, pero antes fue regente y vicedirectora de la escuela y hace 25 años que trabaja como profesora en la escuela. Es profesora de matemática y Licenciada en Gestión y Administración de la Educación.

Dirigir una escuela técnica siendo mujer no es nada fácil.... Estás a prueba todo el tiempo, tenés que demostrar que sabés, que sos fuerte, es que el taller es el centro de una escuela

\footnotetext{
${ }^{3}$ Se resguarda la identidad de los directivos, los nombres no son los reales.
} 
técnica y el taller es un asunto de hombres tradicionalmente. Pero yo hace muchos años que estoy y me respetan ...Yo también trabajaba en uno de los Bachilleres más reconocidos de la Ciudad como profesora y podría haber sido rectora de ahí, pero elegi la escuela técnica y esta escuela... ¿por qué? Porque cuando iba a la sala de profesores de la otra escuela no me gustaba el clima, criticaban a los alumnos, criticaban a todos...y venía acá y veía a los chicos y a los profesores trabajando en el taller y me latía el corazón, me emocionaba ver hacer, ver el aprender haciendo. La elegí con el corazón porque la escuela tiene un corazón enorme. (T1)

La escuela T2 está ubicada en el barrio de Núñez, zona residencial de clase media. Se destaca por su edificio, instalado en una antigua casona de 1914 que supo ser una escuela primaria, que no responde a las necesidades de una escuela técnica. La identidad de la escuela aparece permeada por un discurso históricamente arraigado por el cual hasta hoy se la define en la zona como "el rancho" dado lo pequeño del edificio y cierto deterioro del mismo. Cuenta con 480 estudiantes en tres turnos. El alumnado pertenece a los barrios de emergencia cercanos y también concurren, en menor medida, estudiantes de clase media baja. En los últimos tres años sostiene una tasa de abandono baja y la matrícula inicial es menor a la capacidad de absorción que tiene la escuela. El equipo de conducción está conformado por el Rector, un vicerrector por turno, un Regente, cuerpo de tutores, Jefes de Departamento y una Psicopedagoga.

José es el rector de la Escuela desde hace 2 años y antes ya formaba parte del equipo directivo como Regente en la escuela durante 7 años. Es profesor de física y matemática. Tiene 15 años de antigüedad como docente de la Escuela T1, que forma parte de esta muestra, donde admite que terminó de formarse como docente "en acción”.

Para mi es clave encontrar el sentido de lo que se hace, así encaro la dirección. Nada de burocracia, de esto es lo que manda la normativa...Asísoy como profesor también, primero les pregunto ¿si tuvieran que empujar un auto cómo ponen el cuerpo? Y recién después aparece la trigonometría. En la gestión también trato de llevar a los docentes a la pregunta por el sentido: ¿por qué hacen esto o aquello? ¿Para qué estamos en la escuela? Y me da resultado, acá nadie está en piloto automático. (T2)

En síntesis, si bien los contextos escolares son muy diferentes, los directivos comparten rasgos:

- Formación profesional universitaria y especializada

- $\quad$ Más de 10 años de antigüedad en la escuela.

- Experiencia anterior en cargos directivos

- Fuerte pertenencia institucional y vínculo afectivo con la escuela

\subsection{El liderazgo directivo a partir de las categorías de análisis propuestas}

\section{Visión crítica y reflexiva respecto de las desigualdades y segmentación escolar}

Las cuatro escuelas presentan una población que ha ido cambiando, de la tradicional clase media urbana se pasó a una población estudiantil con fuerte presencia de los sectores vulnerables y los directivos aceptan ese proceso. La visión que predomina en los directivos es la de inclusión genuina y la disminución de las inequidades.

El barrio cambió en los últimos 20 años y esto impactó fuerte en la escuela, hubo que adaptarse... Hay varios sectores sociales, un sector que está mejor, muchos chicos hijos de encargados de edificios de la zona, y hay un sector importante que vive en la villa, pensiones o casas tomadas, y también vienen de la Provincia.... pero esta es una escuela pública, y entonces se entiende que es para todos... Eso es algo que está claro en el proyecto, pero hay que sostenerlo cada día. (C1) 
Tener una población más heterogénea fue un desafío grande para los profesores y hubo que hacer un verdadero cambio de mentalidades en algunos casos. Hablar, hablar y hablar mucho con los docentes y escuchar, escuchar y escuchar a los alumnos. (C2)

Los directivos sostienen una visión crítica con respecto a la dinámica tradicional de escuelas secundarias selectivas y promueven, a través de distintas estrategias, la reflexión colectiva sobre los temas de acceso e inclusión.

Esta es una escuela que tiene una impronta muy tradicional. Fue una escuela de señoritas hasta el año 1970 y de clase media; hoy es mixta y hay chicos de todos los sectores sociales... Cada tanto aparece, en los profesores más antiguos, la idea de que antes era mejor. Y yo les digo mejor no, era distinto. Hoy la escuela secundaria es obligatoria y todos tienen derecho de hacerla. (C1)

Las dos escuelas técnicas destacan por la tradicional estructura selectiva en el diseño original de sus plantas funcionales y su trabajo por disminuir el abandono.

La escuela tiene una estructura piramidal, como todas las secundarias, pero en las técnicas la deserción es más fuerte. O sea, hay 6 ó 7 secciones de primer año y 3 en el último. Ya la estructura es expulsiva. Nosotros venimos bajando el abandono y los últimos años ya son más numerosos. Mi ideal es por supuesto que terminen todos los que empezaron, tener igual cantidad de secciones del primero al último año. (T2)

Antes éramos como toda escuela secundaria un triángulo, empezaban muchosy terminaban pocos, hoy ya somos un trapecio. (T1)

La inclusión de estudiantes con necesidades especiales y embarazo adolescente también configuran una distinción respecto a la normalización vigente en las escuelas secundarias.

Tenemos 25 chicos con necesidades especiales según un acuerdo con la Dirección de Educación especial. Esto hace 20 años atrás era algo impensado. Tienen adaptaciones curriculares y se reciben de Auxiliares de Técnico, pero comparten recreos, educación física y las actividades extraescolares... los psicólogos de la escuela han trabajado mucho con los docentes sobre la inclusión. (T1)

Tenemos chicas embarazadas o que han sido madresy, por supuesto, siguen en la escuela, las apoyamos mucho. (C2)

\section{Proyecto compartido y sentido de comunidad}

Los directivos manifiestan estar liderando un proyecto escolar, con sus particulares características en cada caso, en estrecha relación con la comunidad, lo que se traduce en el hecho de que en tres de las cuatro escuelas la demanda excede la oferta de vacantes en primer año. "Es una escuela buscada" (C2), "Saben que en esta escuela se aprende" (C1), "Pertenecer a la escuela es un motivo de orgullo" (T1), dicen los directivos. Una de las escuelas atraviesa una situación crítica en su edificio y según el director "somos una buena escuela, pero el edificio no ayuda, está en malas condiciones y se están anotando menos chicos en primer año" (T2)

Según los directivos la escuela encarna un sueño de oportunidades para los estudiantes y sus familias. Y se sienten responsables de hacer realidad esa expectativa que pesa sobre la escuela.

Los chicos de menores recursos, como los nuestros, ven a la escuela como un sostén de su presente y el único pasaporte al futuro... Muchos de los padres no fueron a la secundaria o llegaron hasta $2^{\circ}$ o $3^{\circ}$ año y tuvieron que dejar para ir a trabajar. No quieren que pase eso con los chicos... hay mucho compromiso de parte de los padres y confían en la escuela. (T2)

Sabemos que la mayoría tiene familias ensambladas o monoparentales y que muchos de los alumnos trabajan o tienen responsabilidades de cuidado de sus hermanos y de las tareas domésticas...nosotros tenemos que hacer que valga la pena la escuela. (C1)

Los directivos se ocupan de generar un clima escolar de vínculos estrechos entre las personas, cordialidad y confianza. Y esto se registra desde la escuela más pequeña a la más grande. 
Esta es una escuela chica y el contacto es "cara a cara". (T2)

La comunicación en una escuela tan grande de tres turnos necesita ser bien pensada. La tecnología nos permite estar muy bien conectados entre el equipo de conducción y con cada docente. Pero además camino la escuela. (C1)

Somos una dirección de puertas abiertas. (C2)

La relación con los estudiantes es una práctica que los líderes destacan. Existe preocupación común por alentar un clima de convivencia positivo y participativo entre los estudiantes. Todos reconocen que no hay conflictos de convivencia importantes. La existencia y buen funcionamiento de Consejos de Convivencia y de Centros de Estudiantes es valorado por los directivos.

Todos los jueves de 12:15 a 13:30 hs me reúno con el Centro de Estudiantes y siempre hay alguien más, la asesora pedagógica, el Jefe de Talleres o con el arquitecto que es que es maestro de tallery junto con la empresa de mantenimiento se ocupa del edificio y no sólo escuchamos lo que necesitan, nosotros les contamos que proyectos pedagógicos vamos a hacer, cómo va la obra del edificio, y ellos son un canal para el resto de los estudiantes. (T2)

Los líderes se preocupan por establecer relaciones con las familias. "Organizamos tres reuniones en el año, si no vienen los llamamos por teléfono y charlamos individualmente” (C2). En algunos casos trabajan directamente con las familias sobre problemas socioculturales.

Hace unos años a las familias peruanas que ingresaban en primer año (fueron muchos ese año) los tuvimos que reunir y avisarles que para las leyes argentinas el hecho de castigar físicamente a los chicos, pegarles con un cinturón, por ejemplo, no está permitido, que es un delito, que no es normalizar o educar...que si tienen una nota baja no les tienen que pegar. Fue difícil pero la escuela también tiene que trabajar ahí. (T1)

Hay una presencia fuerte de los directivos en la vida cotidiana de la escuela y todos declaran trabajar en equipo. “Acá hay equipo, yo puedo dormir tranquila porque me siento respaldada y confío en ellos” (T2) Vale destacar que en las escuelas públicas las designaciones del personal son individuales, es decir los directivos no conforman sus equipos. Sin embargo, en las escuelas de la muestra se presenta un formato distribuido de liderazgo y se reconocen liderazgos intermedios sólidos. Hay encuentros sistemáticos con distintos actores, sumado a las reuniones informales.

Somos distintos y a veces no estamos de acuerdo, pero las discusiones las hacemos dentro del equipo, una vez que se toma una decisión todos las respaldan. (T2)

Por otra parte, los directivos hablan de todos los adultos de la escuela como parte de un equipo. "El portero es aquí una figura amada por los chicos, en el horario de entrada él está en la puerta y es el primero al que saludan. Él sabe los nombres de todos, quien es amigo de quien, quién anda con problemas” (C2). Se refuerza así la idea de una comunidad que educa y adultos que funcionan como referentes.

Los directivos llevan adelante estrategias diversas para fortalecer la identidad institucional y producir comunidad: el uso de una vestimenta común, la realización de actividades recreativas, las actividades que incluyen a los padres y otros miembros de la comunidad.

Este año armamos un proyecto con toda la comunidad, pintamos el frente para la época de inscripciones, ayudaron los padres y los chicos y chicas y la pintura la compramos con una colecta que hicimos entre los docentes. La idea es que todo lo bueno que tiene la escuela pueda verse desde afuera, para atraer más matrícula también. (T2)

\section{Altas expectativas y compromiso pedagógico}

Los directivos sostienen altas expectativas respecto de los estudiantes, están convencidos de que todos pueden terminar la secundaria y creen que la mayoría va a continuar estudios superiores. "Estos chicos tienen una enorme fortaleza, a pesar de todos los problemas que tienen en sus casas, estudian, trabajan mucho en la escuela" (C2) De la misma manera, manifiestan tener altas expectativas respecto del equipo docente y señalan que la escuela se apoya en los docentes como principal fortaleza. 
Tenemos docentes de lujo, los de mucha antigüedad son pilares y a los nuevos enseguida logramos meterlos en la cultura institucional. (C1)

Aquí hay docentes de muchos años, de gran pertenencia, nos conocemos mucho y hay gran colaboración entre ellos. (C2)

Las expectativas sobre los estudiantes se sostienen aún después de la escuela:

La mayoría acá quiere estudiar en la universidad, para Contador, Economía, entonces los llevamos a que vean clases en la Universidad de Buenos Aires, vuelven entusiasmados. (C1)

También hay expectativas sobre los egresados de quienes hacen un seguimiento. Tenemos un Observatorio de Egresados, porque nos interesa saber dónde y cómo se insertan luego de que terminan la escuela y también sigo de cerca las pasantías laborales que hacen...El otro día en la Feria de Ciencias y Tecnología alguien viene y me felicita por los chicos nuestros que hacen la pasantía en el Centro de Cómputos del Gobierno de la Ciudad...les encanta cómo trabajan...en IBM ya dejaron a 3 egresados como empleados definitivos...eso es una satisfacción. (T2)

El ausentismo docente existe, pero los directivos comparten en que se limita a algunos docentes en particular.

El ausentismo no es un problema, son unos pocos los que faltan, pero igual tenemos un sistema de actividades compensatorias que cada trimestre los docentes deben presentar, de manera que si alguien falta, los chicos tienen un trabajo para hacer. (T2)

El ausentismo de los estudiantes es bajo y allí hay un seguimiento personalizado en las cuatro escuelas.

Sabemos que el ausentismo puede ser la antesala del abandono, por eso tenemos una alerta temprana, llamamos a los padres o el Equipo de Orientación va a la casa. (C1)

La gestión pedagógica aparece como prioritaria en el discurso de los directivos. En las entrevistas el tema académico está presente en forma permanente. Hablan de los aprendizajes y sus problemas, la enseñanza, los proyectos didácticos, la evaluación. Los directivos otorgan relevancia a la dimensión pedagógica y conforman equipos para la gestión pedagógica específica.

Mi prioridad es sostener el foco en lo pedagógico, pero cuesta con tanto trabajo administrativo $y$ atender a las familias. Ahora estoy abocada con los Jefes de Departamento al tema de la evaluación...porque ahí es donde están las prácticas más tradicionales y difíciles de remover.

Garantizar un clima de trabajo, ordenado, tranquilo y a la vez creativo, es una preocupación común. También es común el deseo de tener más tiempo para observar clases.

Este año acordamos algunos criterios comunes para las evaluaciones...me gustaría hacer el seguimiento en forma personal pero no me alcanza el tiempo, la asesora pedagógica es la que se está encargando de ese trabajo que encaramos como una investigación. (C1)

Ante la falta de tiempo de los directivos para el trabajo pedagógico se asegura a través de otros actores y de la observación de otras prácticas:

Nosotros establecimos que sean los docentes los que nos pidan que observemos sus clases, $y$ no decimos observación sino acompañamiento. Yo no puedo ir a todas, pero lo hacen las vicerrectoras. (C2)

No tengo tiempo para hacer observaciones de una clase completa de 80 minutos, pero recorro el taller y me detengo a ver algún trabajo, estoy algunos días a la entrada y los veo llegary hasta observé talleres que arman los tutores. (T1)

En cuanto al desarrollo profesional de los docentes, los directivos lo consideran relevante si bien reconocen que no existen demasiados incentivos externos en el sistema público para el desarrollo profesional:

Tenemos algunos profesionales que no son docentes, sobre todo en las Orientaciones de Informática y Construcciones y generamos nosotros talleres para trabajar aspectos 
didácticos. Tengo la suerte de que la asesora pedagógica y la vicedirectora del turno tarde tienen formación universitaria específica. (T2)

Existen prácticas de retroalimentación interna y colaboración entre docentes que potencian las capacidades. "Los profesores que hacen cursos fuera de la escuela tienen un espacio para socializar lo que aprenden y eso enriquece a todos” (C1). Reconocen que, en ese sentido, la escuela funciona en sí misma como un ámbito de formación.

\section{Prácticas de promoción de la inclusión y la equidad}

Se recoge un repertorio diverso de prácticas proactivas coherentes con la visión crítica referidos en el punto a) que, según los líderes sirven para mitigar y prevenir, dentro del contexto de la propia escuela, las situaciones de inequidad.

Un problema mencionado por todos es la tendencia a la diferenciación socioeconómica por turnos. Tradicionalmente los turnos de la mañana concentran a estudiantes que tienen familias con mayor capital cultural. Para evitar este sesgo y garantizar la inclusión en grupos no segmentados internamente, las escuelas asignan los turnos por sorteo. La escuela T1 implementó una estrategia deliberada de promoción del turno tarde de manera de hacerlo más atractivo, una suerte de "discriminación positiva” para los chicos del turno tarde.

Tratamos por todos los medios de que no haya esa diferencia social entre los turnos. Cuando implementamos los Campamentos hace tres años, tomé la decisión de que comiencen en el turno tarde. Otra, El Bachillerato Internacional va a empezar el año que viene y va a empezar como una oferta en el turno tarde...Justamente para que esa diferencia que es de libro deje de existir y que los mismos padres se convenzan. (T1)

Este mismo problema es abordado por la escuela C1 a través de la conformación de equipos deportivos con integrantes de turno mañana y turno tarde mezclado. Y también a través de actividades recreativas compartidas entre la mañana y la tarde.

Hacen ajedrez, vóley, tenemos una banda y el Club de Ciencias, todo funciona en el entre turno para que se junten. (C1)

Por otra parte, la organización de los grupos de cada sección es materia de una intervención deliberada por parte de la escuela T2 para evitar que se produzcan sesgos y segmentaciones como por ejemplo que en uno de los grupos se concentren los repetidores o los chicos que viven en la villa; la idea es tratar de obtener grupos no segmentados.

La asesora y el psicólogo se entrevistas con cada chico que ingresa y van conformando los grupos de primer año en un trabajo artesanal...no queremos que nadie empiece con etiquetas por pertenecer al grupo tal o cual. (C1)

En las escuelas técnicas se plantea el riesgo de segmentación interna por Orientación. Para evitarlo los directivos implementas acciones deliberadas centradas en proyectos conjuntos, entre ellos proyectos curriculares interdisciplinarios.

Los estudiantes de Informática y los de Mecánica son distintos ... Los de Informática vienen de traje al acto de colación, los otros no. No queremos que sean iguales, pero sí que sientan que son igualmente importantes en la escuela y fuera de ella...lo que hacemos es trabajar por proyectos conjuntos y hay un momento del proyecto que los unos sin los otros no lo pueden resolver. (T1)

La cuestión de género está muy presente en ambas escuelas técnicas. Los directivos señalan que la población de alumnas fue creciendo, pero no pasa el $30 \%$.

Armamos talleres sobre Género para que los docentes puedan trabajar sus prejuicios y poder ver a una alumna mujer tan capaz como a un varón y exigirle lo mismo. (T1)

Existen estudiantes que transitan situaciones judiciales (ya sea por hechos delictivos, violencia doméstica, abusos) y la escuela debe reportar a la Defensoría sobre su desempeño. Algunas escuelas como la C1 tienen más casos que el resto y la dirección también despliega una estrategia específica: 
Tenemos un protocolo que excede lo que nos pide la Defensoría y hacemos un seguimiento bien personalizado a cargo de nuestro Departamento de Orientación, sabemos que la inserción de esos estudiantes en la escuela no es la misma que la de otros y por eso les damos un soporte diferente. (C1)

Todas las escuelas realizan actividades de apoyo y seguimiento personalizado a los estudiantes que presentan mayores dificultades para el aprendizaje. Las estrategias seguidas son: clases de apoyo, ayuda entre pares, actividades de recuperación, previas por parciales (un mecanismo que permite evitar la repitencia fragmentando los exámenes de las materias que quedan pendientes). También, ante situaciones que requieren de ayudas económicas para afrontar por ejemplo salidas didácticas, los directivos disponen el uso de recursos institucionales y de la cooperadora para que todos los estudiantes puedan participar.

\section{Discusión y conclusiones}

Los resultados han permitido alcanzar una comprensión del liderazgo directivo en estas cuatro escuelas que sintetizamos a continuación:

Las escuelas presentan características diversas en cuanto a tamaño, cantidad de estudiantes y docentes, localización e infraestructura. Las problemáticas que se presentan en cada una condicionan las prácticas de liderazgo poniendo énfasis en distintas prioridades y agendas temáticas diversas.

Los perfiles de los directivos comparten rasgos:

- Formación profesional universitaria y especializada en gestión.

- Más de 10 años de antigüedad en la escuela.

- Experiencia anterior en cargos directivos.

- Fuerte pertenencia institucional y vínculo afectivo con la escuela.

El liderazgo directivo evidencia prácticas dentro de las cuatro categorías estudiadas:

- Visión crítica y reflexiva sobre el contexto, con sensibilidad respecto de los problemas de desigualdad, exclusión y segmentación.

- Proyecto compartido y producir un sentido de comunidad, promoviendo un clima escolar de vínculos estrechos y confianza mutua a la vez que trabajar en equipo y desarrollar liderazgo distribuido.

- Altas expectativas respecto de estudiantes y profesorado y foco en los aspectos pedagógicos generando de manera indirecta un clima favorable al aprendizaje.

- Promoción de la equidad y la inclusión a partir de prácticas efectivas y sistemáticas, apoyo a los estudiantes más desaventajados, seguimiento de los aprendizajes e innovaciones contextualizadas.

En concordancia con lo que la literatura internacional señala, los resultados presentados confirman que el factor director aparece como un componente esencial en el desarrollo de buenas prácticas en entornos desfavorables (Leithwood et al., 2004; Ward et al., 2015). Estos directivos se preocupan especialmente por generar un clima escolar que resulte académicamente estimulante, por el aprendizaje de los estudiantes, por generar una cultura de altas expectativas y por fomentar prácticas que promueven el acceso, la inclusión y la equidad a través de liderazgos distribuidos.

Al igual que otras investigaciones realizadas en escuelas con buenas prácticas situadas en contextos vulnerables (Morales, 2017; Tapia-Gutiérrez et al., 2011), encontramos que la creación de entornos de trabajo saludables, donde prevalece una fuerte preocupación por el personal, constituyen herramientas de liderazgo potentes para el desarrollo de buenas escuelas, donde impera un clima de confianza y alta valoración directiva. En una investigación conducida en México, se encontró por ejemplo que lo que marca la 
diferencia entre escuelas de alto y bajo logro académico son justamente las altas expectativas y creencias positivas de los directivos (Morales, 2017). Allí se constató que los directivos de escuelas de bajo rendimiento, en cambio, suelen responsabilizar a los docentes, al sistema educativo y al contexto por los malos resultados, etc., en vez de mantener una mirada positiva hacia la labor docente.

La prácticas de liderazgo encontradas en este estudio definidas como no burocratizadas, de "puertas abiertas", promoviendo relaciones "cara a cara", con presencia fuerte y cotidiana de los directivos que "caminan la escuela", coinciden también con los hallazgos de investigaciones desarrolladas en escuelas en sectores de pobreza de Chile, en las que se constató también que la accesibilidad y disposición de los líderes se conciben como fundamentales: su movilidad "en terreno", en contraposición al trabajo en escritorio, aparece como un valor importante (Bellei et al., 2015).

Los aspectos pedagógicos son el foco de atención y existe un fuerte sentido de responsabilidad por los resultados por parte de los líderes escolares estudiados. Si bien algunos directivos declaran no contar con suficiente tiempo para involucrarse más en la gestión pedagógica y realizar observaciones de clase sistemáticas, fenómeno que coincide con la situación general de los directivos en la Ciudad (Romero y Krichesky, 2019), lo cierto es que en las cuatro escuelas prevalece una preocupación explícita por sostener un liderazgo pedagógico garantizando un ambiente ordenado y seguro que habilite el desarrollo de las tareas académicas lo que confirma resultados de estudios internacionales sobre el tema (Robinson, Lloyd y Rowe, 2014).

Sin embargo, también algunos resultados presentan diferencias con estudios anteriores. En comparación con el reciente estudio sobre los directivos en Argentina realizado por Romero y Krichesky (2019) donde se estudia la normativa, formación y condiciones laborales, el perfil de los directivos estudiados resulta particular ya que se aparta de la media por estar más calificado, contando con formación de postgrado específica y también por presentar antecedentes particulares como la antigüedad en cargos directivos y la pertenencia a la propia escuela.

De la misma manera, el liderazgo pedagógico y proactivo de los directivos de la muestra estudiada contrasta con estudios en el país. En una investigación reciente realizada en la Ciudad de Buenos Aires en la que se aplicó el instrumento TALIS sobre una muestra de 82 directivos de escuelas secundarias públicas y privadas se halló un particular estilo de liderazgo denominado "liderazgo interactivo y reactivo" donde las prácticas de liderazgo y el uso del tiempo está destinado a interacciones con estudiantes y otros actores no docentes más al servicio de "apagar incendios" o controlar conflictos de manera directa, es desmedro de una agenda explicita vinculada al trabajo con docentes y a la generación indirecta de un clima de aprendizaje (Romero y Krichesky, 2018) Los hallazgos aquí presentados contrastan fuertemente con ese estilo y presentan, en cambio, un liderazgo fuertemente proactivo y centrado en la dimensión pedagógica.

No hemos registrado resultados en torno a la existencia de buena infraestructura y equipamiento como factores asociados a las prácticas de liderazgo directivo eficaz y equitativo, muy por el contrario, los directivos de la muestra gestionan en un contexto de recursos limitados y en algún caso de infraestructura muy deficiente.

A modo de conclusión afirmamos que las políticas educativas de lucha contra la inequidad requieren tener en cuenta el factor director como una condición fundamental para el desarrollo de buenas escuelas, capaces de superar las barreras del contexto. Los directores pueden ser considerados además como “informantes cualificados" (Weinstein, Muñoz y Rivero, 2018) de estas políticas educativas y brindar información para anticipar los probables obstáculos de implementación.

En América Latina en general y en Argentina en particular la política de desarrollo del liderazgo es escasa y poco sistemática (Weinstein, Muñoz y Hernández, 2014). En este sentido, esta investigación se propone como un aporte para líneas concretas de políticas, entre las que señalamos:

- Promover una formación directiva centrada en una perspectiva crítica y de cambio, que garantice desarrollo de capacidades para ejercer prácticas de liderazgo pedagógico, distribuido e inclusivo de acuerdo a cada contexto escolar, mejorando los grados de eficacia y equidad.

- Garantizar la constitución de verdaderos equipos directivos de tiempo completo, preparados para el trabajo en contextos vulnerables. Actualmente la designación es individual, a tiempo 
parcial y a elección del directivo sin condición de tener preparación específica para estos contextos,

- Establecer un sistema de incentivos para fomentar el desarrollo profesional de directivos y profesorado que trabaja en escuelas de contextos vulnerables.

- Fomentar y profundizar la investigación sobre el liderazgo directivo eficaz y equitativo.

Esta investigación tiene como limitación el estar basada en las concepciones y percepciones de los propios directivos. De manera complementaria sería interesante realizar a futuro una mayor indagación de sus perfiles con enfoque biográfico narrativo y, para un análisis exhaustivo de agenda, podría ser valioso realizar estudios del tipo shadowing. Del mismo modo, valdría realizar estudios comparativos con directivos de escuelas con estudiantes de similares condiciones socioeconómicas pero bajos resultados con el fin de analizar los contrastes.

Sin dudas, queda mucho por conocer acerca de los modos en que los proyectos educativos pueden operar ampliando los niveles de calidad y equidad y mitigar o prevenir la segregación escolar. Es necesario generar conexiones más estrechas entre la investigación, la mejora de la escuela y el diseño de políticas educativas, de modo que los conocimientos generados tengan un mayor nivel de aplicación en la práctica, desde un enfoque que privilegie los problemas del contexto. De ese modo y tal como señala Reynolds (2012), la implicación de la eficacia escolar en el diseño de políticas públicas puede hacer de ellas entidades más efectivas y, por ende, más justas.

\section{Referencias}

Ainscow, M. y Kugelmass, J. (2005). Leading inclusive schools: A comparison of practices in three countries. Journal of Research in Special Needs Education, 4(3), 3-12.

Arcidiácono, M. (2014). La segregación escolar público-privada en América Latina. CEPAL.

Belavi. G. y Murillo, F. J. (2020). Democracia y justicia social en las escuelas: Dimensiones para pensar y mejorar la práctica educativa. REICE. Revista Iberoamericana sobre Calidad, Eficacia y Cambio en Educación, 18(3), 5-28. https://doi.org/10.15366/reice2020.18.3.001

Bolívar, A. (1999). Como mejorar los centros educativos. Síntesis.

Bolívar, A. (2012). Políticas actuales de mejora y liderazgo educativo. Aljibe.

Bolívar, A. (2019). Una dirección escolar con capacidad de liderazgo pedagógico. La Muralla.

Bolívar, A. y Murillo, F. J. (2017). El efecto escuela: Un reto de liderazgo para el aprendizaje y la equidad. En J. Weinstein y G. Muñoz (Eds.), Mejoramiento y liderazgo en la escuela. Once miradas (pp. 71-112). CEDLE.

Carvallo, M. (2006). Factores que afectan el desempeño de los alumnos mexicanos en edad de educación secundaria: un estudio dentro de la corriente de eficacia escolar. REICE. Revista Electrónica Iberoamericana sobre Calidad, Eficacia y Cambio en Educación, 4(3), 30-53.

Day, C. y Gu, Q. (2016). Educadores resilientes, escuelas resilientes: Construir y sostener la calidad educativa en tiempos difíciles. Narcea.

https://doi.org/10.32541/recie.2016.v1i1.pp122-123

Day, C. y Sammons, P. (2011). Succesfull school leadership linking with learning and achievement. McGraw Hill.

Field, S., Kuczera, M. y Pont, B. (2007). No more failures: Ten steps to equity in education. OECD.

https://doi.org/10.1787/9789264032606-en

Hargreaves, A. y Fullan, M. (2012). Professional capital. Transforming teaching in every school. Teachers College Press.

Krüger, N. (2019a). La segregación por nivel socioeconómico como dimensión de la exclusión educativa: 15 años de evolución en América Latina. Archivos Analíticos de Políticas Educativas, 27(8)

http://doi.org/10.14507/epaa.27.3577 
Krüger, N. (2019b). Segregación escolar: ¿Cuán heterogéneas son las escuelas argentinas? Observatorio Argentino por la Educación.

Leithwood, K., Day, C., Sammons, P., Harris, A. y Hopkins, D. (2006). Successful school leadership: What it is and how it influences pupil learning. Department for Education and Skills.

Leithwood, K., Seashore Louis, K., Anderson, S. y Wahlstrom, K. (2004). How leadership influences student learning. Review of research. Wallace Foundation.

Louis, K. S. (2017). Liderazgos y aprendizajes: Implicancias para la efectividad de las escuelas. En J. Weinstein y G. Muñoz (Eds.), Mejoramiento y liderazgo en la escuela. Once miradas (pp.150-181). CEDLE.

Montoya, S. (2013). Visión para la acción: Los índices de calidad y equidad porteños. Universidad Católica Argentina.

Morales, A. (2017). Aspectos psicosociales y gestión de la evaluación en secundarias de alto y bajo logro. Alteridad, 12(1), 92-103 https://doi.org/10.17163/alt.v12n1.2017.08

Mortimore, P. (1991). School effectiveness research: Which way all the Crossroads? School Effectiveness and School Improvement, 2(3), 213-229. https://doi.org/10.1080/0924345910020304

Murillo, F.J. (Coord.). (2007). Investigación iberoamericana sobre eficacia escolar. Convenio Andrés Bello.

Murillo, F. J. (2008). Enfoque, situación y desafíos de la investigación sobre eficacia escolar en América Latina y el Caribe En R. Blanco (Coord.), Eficacia escolary factores asociados en América Latina y el Caribe (pp. 17-47). UNESCO- LLECE.

Murillo, F.J.y Hernández, R. (2014) Liderando escuelas justas para la justicia social. Revista Internacional de Educación para la Justicia Social, 3(2), 13-32. https://revistas.uam.es/riejs/article/view/337

Murillo, F. J. y Martínez-Garrido, C. (2017). Segregación social en las escuelas públicas y privadas en América Latina. Educacâo \& Sociedade, 38(140), 727-750. https://doi.org/10.1590/es0101-73302017167714

Murillo, F. J. Krichesky, G. J., Castro, A. M. y Hernández, R. (2010). Liderazgo para la inclusión escolar y la justicia social. Aportaciones de la investigación. Revista Latinoamericana de Educación Inclusiva, 4(1), 169-186.

Pereyra, A. (2008). La fragmentación de la oferta educativa en América Latina: La educación pública vs la educación privada. Perfiles Educativos, 30(120), 132-146.

Reynolds, D. (2012). Thinking the unthinkable? The future of school effectiveness and school improvement to be realized through closer relationships with educational policies and policy makers. En C. Chapman, P. Armstrong, A. Harris, D. Muijs, D. Reynolds y P. Sammons (Eds.), School effectiveness and improvement. Research, policy and practice. Challenging the orthodoxy? (pp. 205- 219). Routledge.

Robinson, V. (2017). Hacia un fuerte liderazgo centrado en el estudiante: Afrontar el resto del cambio. En J. Weinstein (Ed.), Liderazgo educativo en la escuela: Nueve miradas (pp. 45-80). Ediciones Universidad Diego Portales.

Robinson, V., Lloyd, M. J., Rowe, K. (2014). El impacto del liderazgo en los resultados de los estudiantes: Un análisis de los efectos diferenciales de los tipos de liderazgo. REICE Revista Iberoamericana de Investigación sobre Cambio y Eficacia Escolar, 12(4e), 13-40.

Román, M. (2013) Factores asociados al abandono y la deserción escolar en América Latina: Una mirada de conjunto. REICE. Revista Iberoamericana sobre Calidad, Eficacia y Cambio en Educación, 11(2), 33-59.

Romero, C. (2018). Hacer de una escuela, una buena escuela. Aique.

Romero, C. (2019). Escuelas resilientes. Estudio sobre factores asociados a logros en escuelas secundarias públicas de gestión estatal de la Ciudad Autónoma de Buenos Aires. En T. Vinacur y L. Landeo (Eds.), Mejora escolar, enfoques, evidencias y experiencias (pp. 57-78). Ministerio de Educación de la Ciudad de Buenos Aires.

Romero, C.y Krichesky, G.J. (2018). Interactive leadership in turbulent school climates. An exploratory study of high school principals from the city of Buenos Aires. Educational Management Administration \& Leadership, 26(2), 339-354. https://doi.org/10.1177/1741143217720456 
Romero, C. y Krichesky, G. (2019). El director escolar en Argentina: Un actor clave, pero (aún) invisible. Un estudio sobre las normas, condiciones laborales y formación de los alumnos escolares de escuelas públicas. Archivos Analíticos de Políticas Educativas, 27(12), art 2. http://doi.org/10.14507/epaa.27.3576

Romero, C., Krichesky, G. y Zacarías, N. (2012) Problemas de justicia social en el contexto educativo argentino. El caso del nivel secundario. Revista Internacional de Educación para la Justicia Social, 1(1), 94-110.

Ryan, J. (2006). Inclusive leadership and social justice for schools. Leadership and Policy in Schools, 5, 3-17. https://doi.org/10.1080/15700760500483995

Ryan, J. (2016). Un liderazgo inclusivo para las escuelas. En J. Weinstein (Ed.), Liderazgo educativo en la escuela. Nueve miradas (pp. 131-148). Ediciones Universidad Diego Portales.

Spillane, J. P. (2006). Distributed leadership. Jossey-Bass.

Spillane, J. P. (2016). Perspectiva distribuida del liderazgo y la gestión escolar: Implicancias cruciales. En J. Weinstein (Ed.), Liderazgo educativo en la escuela. Nueve miradas (pp. 93-129). Ediciones Universidad Diego Portales.

Tapia-Gutiérrez, C., Becerra-Peña, P., Mansilla-Sepúlveda, S. y Saavedra-Muñoz,J. (2011). Liderazgo de los directivos docentes en contextos vulnerables. Educación y Educadores, 14(2), 389-409. https://doi.org/10.5294/edu.2011.14.2.8

Taylor, S. J. y Bogdan, R. (1990). Introducción a los métodos cualitativos de investigación. Paidós.

Vázquez, E. (2012). Segregación escolar por nivel socioeconómico: Midiendo el fenómeno y explorando sus determinantes. CEDLAS.

Ward, S. C., Bagley, C., Lumby, J., Woods, P., Hamilton, T. y Roberts, A. (2015) School leadership for equity: Lessons from the literature. International Journal of Inclusive Education, 19(4), 333-346. http://doi.org/10.1080/13603116.2014.930520

Weinstein, J. (2017). Las escuelas que queremos, los directores que necesitamos. En C. Romero, (Comp.), Ser director (pp. 101-124). Aique.

Weinstein, J., Muñoz, G. y Hernández, R. (2014) El liderazgo escolar en América Latina y el Caribe. Un estado del arte en base a ocho sistemas escolares en la región. OREALC/UNESCO.

Weinstein, J., Muñoz, G. y Rivero, R. (2018). Los directivos escolares como informantes cualificados de las políticas educativas. Sus opiniones bajo el gobierno de Michelle Bachelet en Chile (2014-2017). REICE. Revista Iberoamericana sobre Calidad, Eficacia y Cambio en Educación, 16(3), 5-27.

https://doi.org/10.15366/reice2018.16.3.001

\section{Breve CV de la autora}

\section{Claudia Romero}

Doctora en Educación por la Universidad Complutense de Madrid. Profesora investigadora de tiempo completo del Área de Educación de la Escuela de Gobierno de la Universidad Torcuato Di Tella (UTDT), Argentina. Dirigió el Área de Educación de UTDT y las Especializaciones y Maestrías en Educación desde 2007 a 2020. Es actualmente Directora del Programa de Alta Dirección Escolar de esa universidad y profesora de los posgrados de Políticas Educativas, Administración de la Educación y Políticas Públicas. Es Coordinadora Nacional en Argentina de la Red Iberoamericana de Investigación sobre Cambio y Eficacia Escolar (RINACE) Es Asesora en Educación en organizaciones no gubernamentales y consultora en organismos públicos. Tiene una extensa trayectoria en el trabajo con instituciones educativas del sector público y privado. Es autora de una decena de libros y tiene numerosas publicaciones académicas. Email: claudiaromero@utdt.edu

ORCID ID: https://orcid.org/0000-0001-8416-6486 